\title{
Alternativas de atención odontológica en niños y adolescentes con discapacidad intelectual
}

\section{Resumen}

La discapacidad intelectual, representa alrededor 3 al 5\% de la población mundial, calculándose aproximadamente 400.000 casos en Venezuela. Gran parte de la cual no recibe atención odontológica, a pesar de tener una mayor predisposición a desarrollar caries y enfermedad periodontal. Objetivo: Compartir las herramientas y alternativas para que el odontopediatra pueda abordar de forma individualizada al paciente y tomar en cuenta el grado de discapacidad intelectual. Materiales y métodos: Fuentes de carácter documental de seis países de los cuales 3 son latinoamericanos: España, Estados Unidos, Egipto, Argentina, Colombia y Venezuela entre los años 1992 y 2012. Resultados: Las alternativas de atención odontológica en niños y adolescentes con discapacidad intelectual según lo observado y analizado en la literatura de España, Egipto, Estados Unidos y países latinoamericanos, se basan en técnicas para el acondicionamiento a la consulta odontológica, restricción física, premedicación oral, sedación y anestesia general. Conclusiones: La atención odontológica de niños y adolescentes con discapacidad intelectual se observa con profundas deficiencias ya que son muy pocos los especialistas capacitados y con vocación para atender a este tipo de pacientes, por lo cual se hace necesario la motivación para establecer las alternativas de atención odontológica que servirán de ayuda para los profesionales que deseen profundizar o iniciarse en la adecuada y correcta atención de este tipo de pacientes.

Palabras Clave: discapacidad intelectual, premedicación , sedación, anestesia general.

\section{Artigo Original}

\section{Alternativas de tratamento odontológico em crianças e adolescentes com deficiência mental}

\section{Resumo}

A deficiência mental, representa de 3 al 5\% da população mundial, calculando-se aproxima- damente 400.000 casos na Venezuela. Grande parte não recebe atenção odontológica, apesar de terem uma maior predisposição a desenvolver cáries e enfermidade periodontal. Objetivo:

\footnotetext{
${ }^{1}$ Odontólogo y Especialista en Estomatología del Niño y el Adolescente, Universidad Santa María, Caracas, Venezuela, Magíster en Odontología Hospitalaria y Pacientes Especiales, Universidad de Valencia, España.

${ }^{2}$ Odontólogo; Especialista en Odontología Pediátrica y Doctora en Ciencias "Mención Salud Bucal Materno Infantil" Universidad Central de Venezuela
} 
Compartilhar os recursos e alternativas para que o odontopediatra possa abordar de forma individualizada o paciente e considerar o grau de deficiência mental. Materiais e métodos: Dados de documentos de seis países dos quais três são latinoamericanos: Espanha, Estados Unidos, Egito, Argentina, Colombia e Venezuela entre os anos de 1992 a 2012. Resultados: As alternativas de tratamento odontológico em crianças e adolescentes com deficiência mental de acordo com o observado e analizado na literatura de Espanha, Egito, Estados Unidos e países latinoamericanos, se baseam em técnicas para manejo e adaptação a consulta odontológica, contenção física, premedicação oral, sedação e anestesia geral. Conclusões: Nos tratamento odontológico em crianças e adolescentes com deficiência mental se observa profundas deficiencias uma vez que são poucos os especialistas preparados e com disposição para atender a este tipo de pacientes, pelo qual é necessário motivação para establecer as alternativas de tratamento odontológico que servirão de base para los professionais que desejam se aprofundar ou iniciar de forma adequada e correta no tratamento destes tipos de pacientes.

Palavras Chave: Deficiência Intelectual, pré-medicação, sedação , anestesia geral.

\section{Original article}

\section{Dental care alternatives in children and adolescents with intellectual disability}

\begin{abstract}
The Intellectual disability, representing about 3 to $5 \%$ of the world's population, estimated approximately 400,000 cases in Venezuela. Much of which do not receive dental care, despite having a higher predisposition to develop caries and periodontal disease. Objective: Share tools and alternatives to the dentist can treat the patient individually, taking into account the degree of intellectual disability. Materials and methods: Documentary sources in six countries of which three are in Latin America: Spain,

lescents with intellectual disability as observed and analyzed in the literature of Spain, Egypt, United States and Latin American countries, are based on techniques for conditioning, physical restraint, oral premedication, sedation and general anesthesia. Conclusions: The dental care for children and adolescents with intellectual disability suffers from deep flaws that very few specialists trained and vocation to serve these patients, so it is necessary to establish dental care alternatives that will serve help for professionals who wish to develop or start in the right and proper care for these patients.
\end{abstract} United States, Egypt, Argentina, Colombia and Venezuela between 1992 and 2012. Results: The dental care alternatives for children and ado-
Key Words: Intellectual disability, premedication, sedation, general anesthesia. 


\section{Introducción}

La atención odontológica de los pacientes con discapacidad intelectual, se complica en forma equivalente a la profundidad de la patología de la que son portadores y en especial a su grado de coeficiente intelectual, por lo que el manejo exitoso requiere de consideraciones especiales que el odontólogo debe conocer y aplicar.

En estos pacientes la problemática de la salud es más profunda y compleja que la del resto de la población y la relevancia no viene dada únicamente por la condición bio-psico-social y bucodental de estos niños y adolescentes, sino también por la falta de una adecuada atención odontológica para la demanda creciente que vienen afrontando.

La incidencia de caries, en términos generales, es mayor que en el resto de la población y aumenta de forma progresiva en función de la severidad de la discapacidad intelectual, la complejidad de su manejo y el grado de comunicación; del mismo modo, la enfermedad periodontal destaca por presentar una mayor incidencia y por su aparición a edades tempranas. Las causas se asocian al deficiente mantenimiento de higiene oral, dieta blanda y rica en azúcares, toma de medicamentos, alteraciones estructurales, hábitos parafuncionales o frecuentes traumatismos.

Las maloclusiones son frecuentes y están relacionadas con el síndrome asociado, su gravedad se debe principalmente a su predisposición y a la ausencia de tratamientos. En menor medida se observan también autolesiones, tanto de los tejidos orales como del resto del cuerpo.

Es por esto que en la presente revisión se estudiarán las diferentes alternativas de atención odontológica de los niños y adolescentes con discapacidad intelectual, con la finalidad de proporcionar las herramientas a los profesionales que deseen profundizar o iniciarse en la atención de este tipo de pacientes a objeto de brindar una adecuada atención y de esta manera buscar nuevos caminos para mejorar la calidad de vida de estos niños y adolescentes con discapacidad y favorecer el progreso de la profesión odontológica.

\section{Definición}

La discapacidad según la Organización Mundial de la Salud (OMS, 2012) y la Clasificación Internacional del Funcionamiento, de la Discapacidad y de la Salud (CIF, 2001), es un término general que abarca las deficiencias, las limitaciones de la actividad y las restricciones de la participación. Las deficiencias son problemas que afectan a una estructura o función corporal; las limitaciones de la actividad son dificultades para ejecutar acciones o tareas, y las restricciones de la participación son problemas para participar en situaciones vitales. Por consiguiente, la discapacidad es un fenómeno complejo que refleja una interacción entre las características del organismo humano y las características de la sociedad en la que vive $\mathrm{v}^{1,2}$.

La Discapacidad Intelectual (DI) según la Asociación Americana de Discapacidades Intelectuales y del Desarrollo (AAIDD, 2010), anteriormente denominada Asociación Americana de Retraso Mental (AARM, 2002); se define como una discapacidad caracterizada por limitaciones significativas en el funcionamiento intelectual y la conducta adaptativa, que abarca muchas habilidades sociales cotidianas y prácticas. Esta discapacidad se origina antes de los 18 años ${ }^{3,4}$. 
Tabla 1. Características de la Discapacidad Intelectual.

\begin{tabular}{|c|c|c|c|c|}
\hline VARIABLE & Leve & Moderado & Severo & Profundo \\
\hline Coeficiente Intelectual & $50-55$ a 70 & $35-40$ a $50-55$ & $20-25$ a 35-40 & Menor de 20-25 \\
\hline $\begin{array}{c}\text { Edad de } \\
\text { Fallecimiento (años) }\end{array}$ & $50-59$ & $50-59$ & $40-49$ & Cerca de 20 \\
\hline$\%$ Población & 89 & 7 & 3 & 1 \\
\hline Nivel Socioeconómico & Bajo & Menos bajo & Sin sesgo & Sin sesgo \\
\hline Nivel Académico & $6^{\circ}$ Grado & $2^{\circ}$ Grado & -------- & ------- \\
\hline Educación & Educable & Adiestrable & $\begin{array}{c}\text { No } \\
\text { Adiestrable }\end{array}$ & $\begin{array}{c}\text { No Adiestrable } \\
\text { Residencia }\end{array}$ \\
\hline Colectividad & Tutelada & Supervisados & $\begin{array}{c}\text { Muy } \\
\text { Supervisados }\end{array}$ \\
\hline
\end{tabular}

Fuente: Odontología en Pacientes Discapacitados, p. 70, de Silvestre, 2004, España

\section{Carácterísticas de los niños y adoles- centes con DI}

Existe una gran variabilidad en las actitudes y comportamientos. Por consiguiente, se han establecido niveles de DI generalmente en función del Coeficiente Intelectual (CI). Pueden especificarse cuatro grados de intensidad: leve, moderado, severo y profundo ${ }^{5}$. (Tabla 1 ).

Ciertamente, la discapacidad que afecta a la población infantil constituye un problema de salud integral considerando el impacto negativo que determina sobre el niño, la familia y la comunidad, es por eso que, el bienestar de estos constituye una de las tareas más importantes de la sociedad ya que, la falta de asistencia médica, es causa de altos niveles de mortalidad, morbilidad y discapacidad ${ }^{6}$.

A pesar de que no existen alteraciones bucales típicas o exclusivas en el niño y adolescente con
DI, ellos están más expuestos a desarrollar patologías bucales con mayor frecuencia que el resto de la población. Esto es debido a que es bastante común una higiene bucal deficiente, una alta ingesta de carbohidratos y medicamentos que favorece el desarrollo de caries y enfermedad periodontal. Por otro lado, el manejo de la conducta dificulta el tratamiento odontológico por lo que muchas veces este no es llevado a cabo, debido al poco conocimiento del especialista de las técnicas adecuadas ${ }^{7,8,9}$.

\section{Alternativas de atención odontológica en niños y adolescentes con DI}

Son medios disponibles, realizar una acción que cubra sus necesidades con formas de aplicación de intensidad y complejidad diversa y progresiva.

Según Silvestre (2004), las técnicas que permiten el manejo y tratamiento clínico odontológico de pacientes con DI son: 
- Manejo conductual, la cual es más utilizada en pacientes con DI Leve o DI Moderado en los que se puede establecer un mínimo grado de comunicación.

- La restricción física, utilizada en cualquier grado de DI que así lo requiera para evitar lesiones durante el tratamiento dental.

- La sedación, empleada en pacientes con DI Moderado o DI Severo incapaces de cooperar y finalmente la anestesia general, la cual debe utilizarse cuando hayan fracasado todas las técnicas anteriores o bien cuando se trate de un tratamiento largo y complejo o en pacientes con DI Profundo ${ }^{5}$.

\section{Técnicas para el Acondicionamiento del paciente con DI}

- Decir, Mostrar y Hacer: Esta técnica enseña al niño, paso a paso, lo que se espera de él en la consulta. Se le explica al paciente, con lenguaje comprensible de acuerdo a su CI, lo que se le va a hacer (Decir), luego se la hace una demostración de los elementos que se van a utilizar (Mostar), y por ultimo se procede a llevar a cabo lo explicado (Hacer) (Clinical Guideline on Behavior Management, 2004) ${ }^{10}$. Según Tejada (2006), cuando existe DI uno de los principales problemas es la pobre respuesta a las instrucciones, no es posible tener una comunicación verbal, puede aprender de mejor manera por medio visual ${ }^{11}$. A través de la técnica decir-mostrarhacer, el mostrar es mejor cuando se trata de niños y adolescentes con DI.

En esta técnica hay que considerar su coeficiente de inteligencia y grado de instrucción alcanzado, se debe hablar con lentitud y términos sencillos, dar una sola instrucción en cada orden y un premio tras cumplirla, y escuchar con atención, ya que hay que tener en cuenta que pueden tener problemas de comunicación, por lo cual es mejor las sesiones $\operatorname{cortas}^{12}$.

- Reforzamiento Positivo: De acuerdo a Furman (2004), consiste en el fortalecimiento de un patrón de comportamiento que aumenta la posibilidad, que en un futuro, se exhiba dicha conducta. El especialista debe recompensar el buen comportamiento del niño en la consulta odontológica, mostrando aprobación de los procedimientos realizados ${ }^{13}$.

Los reforzadores pueden ser de varios tipos; pueden ser reforzadores materiales como las recompensas y premios ante las conductas deseadas, de tipo social como las demostraciones verbales de afecto y no verbales, así como los elogios y pueden ser refuerzos de actividad que suponen la oportunidad de realizar una actividad deseada tras realizar la conducta adecuada ${ }^{5}$. Es muy recomendable este tipo de reforzadores en pacientes con DI ya que incrementa las conductas deseadas.

- Reacondicionamiento: Está indicada en niños con experiencias previas poco agradables. Se utiliza una mezcla de diferentes técnicas para de esa manera lograr una mayor aceptación hacia la consulta odontológica (Clinical Guideline on Behavior Management, 2004) ${ }^{10}$. Esta técnica es recomendada en casos de niños y adolescentes que han sufrido malas experiencias, con el fin de reacondicionarlos lentamente, explicándoles de acuerdo al nivel de DI cada procedimiento pero sin mentirles. Por ejemplo, que el niño con DI y el odontopediatra cuenten hasta cinco, se detienen, permitiendo que el niño levante la mano cuando quiera parar, 
sin dejar de establecer claridad sobre el hecho de que la persona que manda es el odontólogo.

- Modelamiento o Imitación: Consiste en la modificación del comportamiento; a través de ello, el paciente puede familiarizarse con la situación odontológica, observando a otros niños en vivo o a través de un video, con un comportamiento igual al que se espera de él, mostrándole el procedimiento a realizar antes de llevarlo a cabo (Furman, 2006) ${ }^{13}$. Usado en los pacientes con DI en cuya edad mental haya un entendimiento de representaciones simbólicas de su medio ambiente.

- Moldeamiento: Según Tejada (2006) y Ferro (2005), esta técnica es el reforzamiento sistemático e inmediato de aproximaciones a la conducta blanco (conducta que se desea instaurar) hasta que esta aparezca en el repertorio de conducta es decir se instale ${ }^{11,14}$. Por ejemplo si queremos enseñarle a un paciente correctamente la técnica de cepillado, debemos primero especificarle que deseamos que aprenda a cepillarse los dientes de manera adecuada y hacer hincapié en la importancia de un buen cepillado para su salud oral, luego le explicaremos la forma en que debe agarrar el cepillo, una vez que lo sostenga bien lo recompensaremos haciéndole saber que lo hizo apropiadamente, posteriormente le enseñaremos a realizar vibración y barrido en cada zona ordenadamente por cuadrante reforzando cada vez que lo haga adecuadamente y corrigiéndolo cuando se equivoque hasta que el paciente domine correctamente la técnica de cepillado.

- Corrección Física: Consiste en llevar físicamente al paciente a realizar la actividad es- perada, no efectuada por el niño después de una instrucción o corrección verbal. Debe de ir acompañado con cierta fuerza, teniendo especial cuidado de no causar dolor ni lastimar al niño (Tejada, 2006) ${ }^{11}$. Esto se recomienda solo en pacientes con DI leve o moderado.

- Extinción: Como lo menciona Reeve (1994), es la eliminación del reforzador de una conducta que anteriormente se reforzaba. La manera más efectiva de erradicar una conducta reforzada anteriormente es ignorándola y para ello se debe ser constante y persisten$t^{15}$. Un ejemplo de esta técnica lo representa el paciente infantil que llora durante el tratamiento y el operador sin querer refuerza la conducta atendiéndolo, consintiéndolo o diciéndole que no llore, cuando se da cuenta decide utilizar un programa de extinción para disminuir la conducta del llanto, ignorando el llanto, aplicando las siguientes medidas: antes que el niño llore se le realizan preguntas de su interés (mascotas, juguetes, etc.), sigues realizando el procedimiento y actuar como si no oyera los llantos, perseverar hasta lograr la extinción del llanto. Este método en los niños con discapacidad se realiza cuando presentan conductas que se manifiestan con rabietas, pataletas, lloros, pero sin manifestaciones agresivas.

- Reforzamiento de Conductas Incompatibles: Es la disminución de una conducta que es incompatible con la conducta que deseamos modificar. Se aplica reforzando al paciente por cualquier otra conducta diferente a la conducta inapropiada que se quiere modifica ${ }^{16}$. Por ejemplo, al niño que acostumbre agarrarle la mano al operador para intentar retirarla mientras se le está trabajando en boca, se le pide que coloque la mano en la 
barriga y se le refuerza el buen comportamiento diciéndole que es un niño muy obediente por ponerse las manos en la barriga. Esta técnica funciona en pacientes con DI que puedan comprender el significado de los procedimientos dentales y cuya compresión del lenguaje no sea limitada.

- Desensibilización: Longobardi y Martínez en 1997, describen que esta técnica se emplea para reducir los temores y tensión del paciente. Se logra enseñando una respuesta competente como la relajación, y luego se introducen progresivamente estímulos más amenazantes a medida que el paciente se desensibiliza. Se realizan procedimientos fáciles y menos amenazantes dejando los más difíciles para más adelante. Presentando primero el espejo, pinza, conos de goma y luego el explorador, la turbina, jeringa, etc ${ }^{17}$. Se emplea mostrando y poniendo en funcionamiento las partes del equipo dental cuando el paciente tiene una DI leve o DI moderada.

- Restricción Física: Según lo mencionan Silvestre (2004) y Furman (2004), la restricción física supone cualquier método manual, físico o mecánico aplicado al paciente con discapacidad para inmovilizarlo, parcial o totalmente, con el fin de protegerlo a él, al especialista y su equipo mientras se le proporciona atención odontológica. Deberá obtenerse antes de comenzar el tratamiento el consentimiento informado de los padres o responsables del paciente especificando el tipo de inmovilización que se va a realizar. Esta indicada en pacientes incapaces de cooperar debido a su corta edad, inmadurez emocional o discapacidad física o mental y contraindicada en pacientes cooperadores o aquellos donde no hay posibilidad de hacer una inmovilización segura debido a sus condiciones médicas, psicológicas o de desarrollo físico. Nunca debe ser aplicada como castigo, ni de forma exclusiva como conveniencia personal ${ }^{5,13}$. Este es el último recurso, no es recomendable pero en casos de emergencia, la prioridad del tratamiento nos puede llevar a ejecutarla.

- Técnicas de premedicación oral: Además del manejo psicológico del comportamiento del niños y adolescente, y aún con la ayuda de la restricción física, el tratamiento de buen número de pacientes con DI no puede realizarse de manera óptima, debido a que muchos de estos muestran gran resistencia emocional y/o física y una capacidad de comprensión y comunicación limitadas, así como un grado de colaboración escaso. Esto, los hace ser candidatos frecuentes al empleo de fármacos, como las benzodiacepinas, que permitan la relajación del paciente. Tal es el caso de los pacientes con DI Moderado cuya alternativa de atención odontológica es la premedicación oral, además de las anteriormente nombradas (Silvestre, 2004 y Álvarez, 2006) $)^{5,18}$. Siempre en interconsulta con el pediatra o anestesiólogo, ya que se podrían presentar complicaciones médicas que deben ser resueltas interdisciplinariamente.

- Técnicas de Sedación: En muchas ocasiones, cuando el grado de discapacidad a nivel cognitivo es alto y existe falta de comunicación o incapacidad para controlar los movimientos involuntarios, se hará necesario recurrir a la ayuda farmacológica para realizar en las mejores condiciones el tratamiento odontológico. Como lo menciona Silvestre (2004), esta es la esta alternativa de atención odontológica en niños y adolescente con DI Moderado o DI Severo incapaces de cooperar ${ }^{5}$. En Venezuela según la legislación vigente y 
la doctrina imperante en nuestro país, esta técnica sólo puede ser ejecutada por un Médico especialista en Anestesiología. Según, la American Academy of Pediatric Dentistry (AAPD, 1992), los objetivos de la sedación en pacientes pediátricos son: proveer, facilitar y aumentar un buen cuidado del paciente; minimizar los comportamientos extremadamente disruptivos; promover una respuesta positiva al tratamiento odontológico; promover el bienestar y la seguridad del paciente; garantizar que el paciente regrese al estado fisiológico que presentaba antes de la sedación ${ }^{18,19}$.

- Técnicas de Anestesia General: La anestesia general según Casanova (1994), es un estado de pérdida de la conciencia inducido por fármacos durante el cual el paciente no puede ser despertado ni aún con estímulos doloro$\operatorname{sos}^{20}$. Es un estado transitorio, reversible, de depresión del sistema nervioso central inducido por drogas específicas y caracterizado por pérdida de la conciencia, de la sensibilidad, de la motilidad y de los reflejos. En este estado existe analgesia, amnesia, inhibición de los reflejos sensoriales y autónomos, relajación del músculo estriado y pérdida de la conciencia. Habitualmente hay incapacidad para mantener la respiración en forma independiente ya que la droga induce depresión respiratoria, requiriéndose asistencia para mantener la permeabilidad de la vía aérea y presión positiva para permitir la respiración. La función cardiovascular puede estar disminuida. Esta es la técnica indicada en casos donde la colaboración o tolerancia del paciente son casi nulas o la discapacidad psíquica es muy severa, siendo esta la alternativa de atención en niños y adolescentes con DI profunda ${ }^{5,18,20}$. De acuerdo a Guerra
(2003), cuando el tratamiento bucal es efectuado bajo anestesia general o sedación profunda, está a cargo un médico anestesiólogo con entrenamiento en anestesiología pediátrica, quien se encarga de los cuidados preoperatorios que estos pacientes requieren $y$ forma parte del equipo interdisciplinario ${ }^{21}$.

- El protocolo de atención para pacientes que requieren esta modalidad es: preparar al paciente para el monitoreo por medio de oxímetro de pulso, tensiómetro y cardioscopia, tomar una vía en el dorso de la mano para el suministro endovenoso de medicamentos, intubación del paciente, colocación del packing bucofaringeo para la protección del paciente, ya que este impide el paso de restos de material de deshecho dental y de obturación a las vías respiratorias y faringe para posterior realización del tratamiento dental completo $^{22}$.

\section{Objetivo general}

Establecer las alternativas de atención odontológica en niños y adolescentes con Discapacidad Intelectual en base a la literatura de diferentes paises a objeto de brindar una adecuada atención en la consulta odontopediátrica.

\section{Material y métodos}

La presente, es una investigación de tipo documental, considerada dentro del nivel descriptivo porque realiza una descripción con precisión, indicando sus rasgos más peculiares y con un diseño bibliográfico lo cual es básico de las investigaciones documentales ya que a través de la revisión del material documental de manera sistemática, rigurosa y profunda se llega al análisis de diferentes fenómenos. La información 
requerida para abordar esta investigación se encuentra en materiales impresos, audiovisuales y electrónicos, es decir, en fuentes de carácter documental de seis países de los cuales 3 son latinoamericanos: España, Estados Unidos, Egipto, Argentina, Colombia y Venezuela publicados entre los años 1992 y 2012.

\section{Resultados}

Las alternativas de atención odontológica en niños y adolescentes con discapacidad intelectual según lo observado y analizado en la literatura de España, Egipto, Estados Unidos y países latinoamericanos entre los años 1992 y 2012 (ta-

Tabla 2. Aportes bibliográficos de diversos autores por año y país.

\begin{tabular}{|c|c|c|}
\hline AÑO & PAIS & APORTE \\
\hline 1992 & Venezuela & Técnicas de modificación conductual \\
\hline 1994 & $\begin{array}{c}\text { España } \\
\text { Venezuela }\end{array}$ & $\begin{array}{l}\text { Psicología de la conducta } \\
\text { Anestesia General }\end{array}$ \\
\hline 1997 & Venezuela & Condiciones bucales \\
\hline 1999 & $\begin{array}{l}\text { Argentina } \\
\text { Venezuela }\end{array}$ & $\begin{array}{c}\text { Metodología de atención en niños con discapacidad } \\
\text { Prevalencia de patologías bucales }\end{array}$ \\
\hline 2001 & USA & $\begin{array}{c}\text { Clasificación Internacional del funcionamiento de la } \\
\text { Discapacidad y de la Salud }\end{array}$ \\
\hline 2002 & USA & Discapacidad Intelectual \\
\hline 2003 & $\begin{array}{l}\text { Venezuela } \\
\text { Colombia }\end{array}$ & $\begin{array}{l}\text { Anestesia general } \\
\text { Manejo del paciente }\end{array}$ \\
\hline 2004 & $\begin{array}{l}\text { España } \\
\text { USA } \\
\text { Colombia }\end{array}$ & $\begin{array}{l}\text { Odontología en pacientes con discapacidad } \\
\text { Tratamiento odontológico en discapacidad } \\
\text { Manejo del comportamiento } \\
\text { Técnicas de modificación conductual }\end{array}$ \\
\hline 2005 & $\begin{array}{l}\text { Venezuela } \\
\text { USA }\end{array}$ & $\begin{array}{c}\text { Técnicas de modificación conductual } \\
\text { Salud Oral en pacientes con DI } \\
\text { Técnicas Sedación }\end{array}$ \\
\hline 2006 & $\begin{array}{l}\text { Venezuela } \\
\text { Colombia } \\
\text { España }\end{array}$ & $\begin{array}{c}\text { Técnicas de sedación } \\
\text { Sedación Oral } \\
\text { Discapacidad Intelectual }\end{array}$ \\
\hline 2007 & Polonia & Caries y enfermedad periodontal \\
\hline 2010 & USA & Discapacidad Intelectual \\
\hline 2012 & $\begin{array}{c}\text { USA } \\
\text { Egipto }\end{array}$ & $\begin{array}{c}\text { Discapacidad } \\
\text { Caries en niños y adolescentes con DI }\end{array}$ \\
\hline
\end{tabular}


Tabla 3. Resultados: Alternativas de Atención Odontológica en niños y adolescentes con Discapacidad Intelectual.

\begin{tabular}{|c|c|}
\hline $\begin{array}{c}\text { Alternativas de Atención Odontológica en } \\
\text { niños y adolescentes con Discapacidad } \\
\text { Intelectual }\end{array}$ & $\begin{array}{c}\text { Tipo de Discapacidad Intelectual según } \\
\text { su Coeficiente Intelectual }\end{array}$ \\
\hline Técnica de acondicionamiento & DI de leve a Moderado \\
\hline Técnica de Pre-medicación Oral & DI Moderado \\
\hline Técnica de Sedación & DI de Moderado a Severo \\
\hline Técnica de Anestesia General & DI Profundo \\
\hline
\end{tabular}

Fuente: Elaborada por la autora según la conclusión a los diferentes autores estudiados.

bla 2), se basan en técnicas para el acondicionamiento a la consulta odontológica, restricción física, premedicación oral, sedación y anestesia general.

Según los aportes bibliográficos de España basados en discapacidad intelectual, psicología de la conducta, atención al paciente con discapacidad; de Estados Unidos en definición de términos y clasificación de discapacidad intelectual, técnicas de manejo de conducta, técnicas de sedación; de Egipto referente a la presencia de caries en pacientes con discapacidad intelectual y en los países latinoamericanos fundados en técnicas de modificación de conducta, condiciones orales, premediación oral, técnica de sedación y anestesia general se pudieron establecer las diferentes alternativas de atención odontológica en niños y adolescentes con discapacidad intelectual según sus características y de acuerdo al coeficiente intelectual (tabla 3).

\section{Discusión}

La DI constituye un problema mundialmente difundido y se presenta en personas de todas las razas y etnias y es el trastorno más frecuente del desarrollo poniéndose en evidencia desde la infancia, ya que afecta a aproximadamente al $1 \%$ de la población mundial según Torpey, 2002 y según estimaciones de la OMS, el rango es de $3 \%{ }^{23}$.

Según Dávila, Gil, Daza, Bullones y Ugel (2005), los problemas dentales se encuentran entre las diez primeras causas que limitan la actividad de las personas con DI, siendo los mas frecuentes la caries dental y la enfermedad periodontal, que en muchos casos conlleva a la pérdida dental. Un estudio realizado por Manzano y col., concluyó que el 71,33\% de la muestra evaluada presentaba caries dental y un 52,63\% cambio de coloración en la encía ${ }^{24,25}$. Coincidiendo con estos resultados, Dávila y col. estudiaron 4 poblaciones diferentes con DI, donde se determinó que el $64,7 \%$ de los pacientes con DI leve presentaba caries, así como el 64,5\% de los diagnosticados con DI moderado ${ }^{24}$. De acuerdo a Bakrys y Alaki (2012), el tipo de escuela, la dieta y el nivel de función (completamente independiente, completamente dependiente o dependiente en parte de los cuidadores) son factores de riesgo asociados a la presencia de caries en niños y adolescentes con $\mathrm{DI}^{26}$. Por otro lado, Górski y 
col. afirman después de realizar un estudio con 41 pacientes con DI con edades comprendidas entre 15 y 18 años que hay una baja incidencia tratamientos odontológicos efectuados pero una tasa más elevada de pérdida dental ${ }^{9}$.

Como lo refiere Cárdenas (2003), cuando se realiza la adaptación a la consulta es importante tener en cuenta cuales son los objetivos de tratamiento del paciente y determinar la factibilidad de lograrlo con técnicas no farmacológicas, de lo contrario deben considerarse otras alternativas tales como la premedicación oral, la sedación o la anestesia general ${ }^{27}$.

\section{Conclusiones}

La atención odontológica de niños y adolescentes con DI adolece de profundas deficiencias ya que son muy pocos los especialistas capacitados y con vocación para atender a este tipo de pacientes, por lo cual se hace necesario establecer las alternativas de atención odontológica que servirán de ayuda para los profesionales que deseen profundizar o iniciarse en la adecuada y correcta atención de este tipo de pacientes.

En base a las características de los niños y adolescentes con discapacidad intelectual y de acuerdo a su coeficiente intelectual se pueden establecer las alternativas de tratamiento para cada uno de ellos. Los niños y adolescentes con discapacidad intelectual leve van a ser manejados por el odontopediatra a través del abordaje conductual con las diferentes estrategias para el acondicionamiento. En el caso de los niños y adolescentes con discapacidad Intelectual moderado a demás de las técnicas de acondicionamiento se puede utilizar la técnica de premedicación oral y en caso de ser necesario la restricción física. En los pacientes con discapacidad intelectual severo y profundo serán atendidos bajo la técnica de sedación y anestesia general respectivamente.

\section{Referencias}

1. World Health Organization. Temas de Salud. Discapacidades. Organizacion Mundial de la Salud. WHO; 2012.

2. World Health Organization. Clasificación Internacional del Funcionamiento de la Discapacidad y de la Salud. Organización Mundial de la Salud. Geneva: WHO; 2001.

3. Schalock, R., Borthwick-Duffy, S., Bradley, V., Buntix, W.H.E., Coulter, M-D., Craig, E, Gomez, S., Lachapelle, Y., Luckasson, R., Reeve, A., Shogren, K., Snell, M., Spreat, S., Tassé, M., Thompson, J., Verdugo, M., Wehmeyer, M. and Yeager, M. Intellectual Disability. Definition, Classification, and Systems of Supports. 11th Edition. Washington, D.C.: American Association on Intellectual and Developmental Disabilities, 2010.

4. Luckasson, R., Borthwick-Duffy, S., Buntix, W.H.E., Coulter, D.L., Craig, E.M., Reeve, A., y cols. Mental Retardation. Definition, Classification and Systems of supports. 10th Edition. Washington, DC: American Association on Mental Retardation, 2002.

5.- Silvestre, J. Odontología en Pacientes Discapacitados. Valencia: Editorial Laboratorios Kin, S.A., 2004.

6. Ravaglia, C. Odontología en niños discapacitados. Metodología para su atención. Buenos Aires: Fundación Severo Vaccaro, 1999.

7. Contreras, K. El Paciente Impedido. Un enfoque Preventivo de Salud Oral. (Trabajo Especial de Grado no publicado). 1994. Universidad Central de Venezuela. Caracas.

8. Pérez D. Propuesta para la Aplicación de Técnicas de Sedación en la Atención de Pacientes Discapacitados en la Sala Clínica de Postgrado de la Facultad de Odontología de la UCV. (Trabajo de ascenso no publicado). 2006. Universidad Central de Venezuela. Caracas. 
9. Górski M., Buczkowska-Radlińska J. Teeth and periodontium status of moderately mentally retarded children and the health awareness of their parents. Ann Acad Med Stetin 2007; 53(2):92-9.

10. Clinical Guideline on Behavior Management: Pediatric Dentistry Referente Manual 2003-2004. 2004; 25(7), 70.

11. Tejada, M. Retraso Mental de Origen Genético. España. 2006. Documento en línea, obtenible en: http: / / dialnet.unirioja. es / servlet/articulo?codigo=1425335. [Consulta: 2008].

12. Bullón, P. Machuca, G. Tratamiento odontológico en pacientes especiales. 2da Edición. Madrid: Normon; 2004.

13. Furman, E. Hacia la Construcción de una Clínica Agradable para los niños en la Facultad de Odontología de la Pontifica Universidad Javeriana. (Trabajo Especial de Grado). 2004. Pontifica Universidad Javeriana. Colombia.

14. Ferro, M. Técnicas de Modificación Conductual Aplicables en la Clínica Odontológica. Acta Odontológica Venezolana 2005; 43, (2). Venezuela.

15. Reeve, J. Motivación y Emoción. Madrid: McGraw-Hill, 1994.

16. Fraga, J. Técnicas de Modificación de Conducta. (Tesis mimeografiada. Facultad de Humanidades). 1992. Escuela de Psicología de la Universidad Central de Venezuela. Caracas.

17. Longobardi, M. y Martínez, V. Condiciones del Componente Bucal de Salud en un grupo de Pacientes Institucionalizados con Retardo Mental del Área Metropolitana. (Trabajo Especial de Grado no publicado). 1997. Universidad Central de Venezuela. Caracas.

18. Álvarez, A. y Álvarez, M. Sedación Oral: Fundamentos Clínicos para su Aplicación en Odontología. Revista CES Odontología, 2006; 19(2), 61-73.

19. Pisalchaiyong T, Thairatvorakul C, Jirakijja J, Yuktarnonda W. Comparison of the Effectiveness of oral Diazepam and Midazolam for the Sedation of Autistic Patients During Dental Treatment. Pediatric Dentistry, 2005: 27(3).

20. Casanova, M. El Paciente en Desventaja. Una Realidad- Una Propuesta. (Trabajo de ascenso no publicado). 1994. Universidad Central de Venezuela. Caracas.

21. Guerra, M., Tovar, V., Salazar, A. y Garrido, E. Tratamiento odontológico en pacientes pediátricos vih $(+)$ bajo anestesia general en la Facultad de Odontología de la Universidad Central de Venezuela. Acta Odont Venez, 2003; 41(2).

22. Guerra, M., Casanova, M., Suarez, J. y Salazar, A. Tratamiento odontopediatrico de un paciente vih (+) bajo anestesia general; primer caso en el postgrado de odontología infantil en la facultad de odontología de la UCV. Acta Odont Venez, 2003; 41(1).

23. Torpey, J. Retraso Mental. The Journal of the American Medical Association, 2002, September; 288(12). USA.

24. Dávila, M., Gil, M., Daza, D., Bullones, X., Ugel, E. Salud Oral de las personas con Retraso Mental en cuatro Municipios del Estado Lara, 2003. Acta Odont Venez, 2005; 43(3).

25. Pirela, A., Salazar, C., Manzano, M. Patología Bucal Prevalente en Niños Excepcionales. Acta Odont Venez, 1999; 37(3).

26. Bakrys, NS. Alaki, SM. Risk factors associated with caries experience in children and adolescents with intellectual disabilities. J Clin Pediatr Dent. 2012; 36(3):319-23.

27. Cárdenas J. Odontología Pediátrica. Corporación para Investigaciones Biológicas. Colombia: Tercera Edición, 2003.

Recibido: 24-11-2012

Aceptado: 29-12-2012

Correspondencia: mariasofialozada@hotmail.com 